Research Article

\title{
Some Variants of Strong Normality in Closure Spaces Generated via Relations
}

\author{
Ria Gupta $(D)$ and Ananga Kumar Das (iD \\ School of Mathematics, Shri Mata Vaishno Devi University, Katra 182320, Jammu and Kashmir, India \\ Correspondence should be addressed to Ananga Kumar Das; akdasdu@yahoo.co.in
}

Received 6 June 2021; Accepted 28 June 2021; Published 12 July 2021

Academic Editor: Frédéric Mynard

Copyright (c) 2021 Ria Gupta and Ananga Kumar Das. This is an open access article distributed under the Creative Commons Attribution License, which permits unrestricted use, distribution, and reproduction in any medium, provided the original work is properly cited.

In this paper, some variants of strongly normal closure spaces obtained by using binary relation are introduced, and examples in support of existence of the variants are provided by using graphs. The relationships that exist between variants of strongly normal closure spaces and covering axioms in absence/presence of lower separation axioms are investigated. Further, closure subspaces and preservation of the properties studied under mapping are also discussed.

\section{Introduction and Preliminaries}

In 1960, the theory of digital topology arose for the study of geometric and topological properties which in turn can be used in computer graphics, image processing, etc. Digital image processing is a rapidly growing discipline and has many applications in allied branches of mathematics. Rosenfeld [1] in 1979 studied connectedness, thinning, and algorithm for border. To compare digital topology and general topology, the generalized topological structure was introduced in [2] by Smyth. The topological structure that arises from the directed graph is used in digital topology, while Šlapal [3] in 2003 studied closure operations for digital topology. He studied the closure space that arises from $\alpha$-ary relation and also studied connectedness in digital spaces via closure operators on graphs [4]. Closure spaces defined through binary relations were introduced in 2006 [5] by Allam et al., and in 2008 [6], the same authors generated topologies by using relations. They proved that topology generated from aftersets and foresets is dual if the relation is preorder. They also introduced lower separation axioms in terms of relation and studied these closure spaces in digital topology. Šlapal and Pfaltz [7] utilized binary relations in networks and studied closure operators associated with networks. B. M. R. Stadler and P. F. Stadler [8] studied some higher separation axioms in closure spaces, and recently, Gupta and Das [9] investigated variants of normality in closure setting by using cannonically closed sets. In 2018, Gupta and Das [10] introduced higher separation axioms such as strongly normal and strongly regular closure spaces via binary relation.

Let $X$ be any set; then, a relation on $X$ is a subset of $X \times X$, i.e., $R \subseteq X \times X$. The formula $(x, y) \in R$ is abbreviated as $x R y$, which means that $x$ is in relation $R$ with $y$. In 2006, afterset of $x \in X$ was denoted and defined as $x R=\{y: x R y\}$ and foresets were denoted and defined as $R x=\{y: y R x\}$ [6]. Liu [11], in 2010, represented aftersets and foresets as left relation and right relation. A set $\langle p\rangle R$ is the intersection of all aftersets containing $p$, i.e., $\langle p\rangle R=\cap_{p \in x R} x R$, if there exists $x$ such that $p \in x R$. In this paper, we introduced variants of strongly normal closure spaces i.e., normal and almost-normal closure spaces. Some properties of newly defined notions are studied, and the relation between new notions and lower separation axioms is investigated with the help of covering axioms. As most of the practical situation involves binary relation, which can easily be expressed through graphs, an attempt in this paper is made to provide examples via graphs to support notions defined in this paper. 
Definition 1 (see [5]). Let $X$ be any set and $R \subseteq X \times X$ be any binary relation on $X$. The relation $R$ gives rise to a closure operation $\mathrm{cl}_{R}$ on $X$ as follows:

$$
\mathrm{cl}_{R}(A)=A \cup\{x \in X:\langle x\rangle R \cap A \neq \varnothing\},
$$

which satisfies the following conditions:

$$
\begin{aligned}
& \text { (1) } \operatorname{cl}(\varnothing)=\varnothing, \\
& \text { (2) } A \subseteq \operatorname{cl}(A), \\
& \text { (3) } \operatorname{cl}(A \cup B)=\operatorname{cl}(A) \cup \operatorname{cl}(B)
\end{aligned}
$$

In addition to the above three properties, it also satisfies the idempotent condition. The set along with the operator $\mathrm{cl}_{R}$ is a closure space. In the closure space $\left(X, \mathrm{cl}_{R}\right)$, a set $A$ is closed [5] if $\mathrm{cl}_{R}(A)=A$.

Lemma 1 (see [5]). Let $R$ be any binary relation on a nonempty set $X$; then, $\{x\} \cup\langle x\rangle R$ is a minimal neighborhood of $x$ for all $x \in X$ i.e., $N_{R}(x)=\{x\} \cup\langle x\rangle R$.

Remark 1 (see [5]). The minimal neighborhood of a point $x$ in a closure space $\left(X, \mathrm{cl}_{R}\right)$ is defined as follows:

$$
N_{R}(x)= \begin{cases}\langle x\rangle R, & \text { if }\langle x\rangle R \neq \varnothing, \\ \{x\}, & \text { if }\langle x\rangle R=\varnothing .\end{cases}
$$

Lemma 2 (see [5]). For any binary relation $R$ on $X$ if $x \in\langle y\rangle R$, then $\langle x\rangle R \subseteq\langle y\rangle R$.

Theorem 1. In a closure space $\left(X, c l_{R}\right), c l_{R}\left(\cup N_{R}(x)\right)$ is the smallest closed set containing $N_{R}(x)$.

Definition 2. In a closure space $\left(X, \mathrm{cl}_{R}\right)$, a set $A$ is said to be regularly closed if for a closed set $\mathrm{cl}_{R}(A)=A, \mathrm{cl}_{R}\left(\right.$ int $_{R}$ $(A))=A$, and a set is said to be regularly open if its complement is regularly closed, i.e., $\operatorname{cl}_{R} \operatorname{int}_{R}\left(X-\mathrm{cl}_{R}\right.$ $(A))=X-\mathrm{cl}_{R}(A)$.

Definition 3 (see [5]). A closure space $\left(X, \mathrm{cl}_{R}\right)$ generated from a binary relation $R$ is said to be $T_{1}$ if and only if for every two distinct points $x$ and $y \in X$, both $x \notin\langle y\rangle R$ and $y \notin\langle x\rangle R$ hold.

Definition 4 (see [5]). Let $R$ be any binary reflexive relation; then, a closure space $\left(X, \mathrm{cl}_{R}\right)$ generated from $R$ is called as a $T_{2}$-space if and only if for every two distinct points $x, y \in X\langle x\rangle, R \cap\langle y\rangle R=\varnothing$.

Definition 5 (see [10]). Let $R$ be a binary relation on $X$; then, the closure space $\left(X, \mathrm{cl}_{R}\right)$ is said to be strongly normal if for two disjoint closed sets $A=\mathrm{cl}_{R}(A)$ and $B=\mathrm{cl}_{R}(B)$, there exist distinct $x$ and $y$ such that $A \subseteq(\langle x\rangle R), B \subseteq(\langle y\rangle R)$, and $\langle x\rangle R \cap\langle y\rangle R=\varnothing$.

Definition 6 (see [10]). Let $R$ be a binary relation on $X$; then, the closure space $\left(X, \mathrm{cl}_{R}\right)$ is said to be strongly regular if for any closed set $\mathrm{cl}_{R}(A)=A$ and a point $x \notin \mathrm{cl}_{R}(A)$, there exist disjoint $\langle u\rangle R$ and $\langle v\rangle R$ such that $x \in\langle u\rangle R$ and $\mathrm{cl}_{R}(A) \subseteq\langle v\rangle R$.

\section{Variants of Strongly Normal Closure Space}

Definition 7. Let $R$ be a binary relation on $X$; then, the closure space $\left(X, \mathrm{cl}_{R}\right)$ is said to be normal if for two disjoint closed sets $\mathrm{cl}_{R}(A)=A$ and $\mathrm{cl}_{R}(B)=B$, there exist some $x$ and $y$ in $\operatorname{cl}_{R}(A)$ and $\operatorname{cl}_{R}(B)$, respectively, such that $\mathrm{cl}_{R}(A) \subseteq \cup_{x \in A} N_{R}(x)=U, \quad \mathrm{cl}_{R}(B) \subseteq \cup_{y \in B} N_{R}(y)=V$, and $U \cap V=\varnothing$.

The following example establishes that there exists a closure space generated from a relation which is normal but not strongly normal.

Example 1. Let us consider the set $X$ consisting of straight lines in a plane and define a binary relation $R$ on $X$ as $a R b$ if and only if line $a$ is parallel to line $b$. Clearly, the closure space $\left(X, \mathrm{cl}_{R}\right)$ is normal but not strongly normal because for two disjoint closed sets, there do not exist disjoint $\langle x\rangle R$ and $\langle y\rangle R$ containing them.

Example 2. A closure space generated from a graph which is not normal.

From the directed graph in Figure 1, we have $a R=\{c, d\}, b R=\{a, b, c\}, c R=\{b, c\}$, and $\mathrm{d} R=\{c\}$. Then, $\langle a\rangle R=\{a, b, c\},\langle b\rangle R=\{b, c\},\langle c\rangle R=\{c\}$, and $\langle d\rangle R=\{c, d\}$. The closure space $\left(X, \mathrm{cl}_{R}\right)$ is not normal because for two disjoint closed sets $\operatorname{cl}_{R}(A)=\{a\}=A$ and $\operatorname{cl}_{R}(B)=\{d\}=B$, there does not exist some $x$ and $y$ such that $\operatorname{cl}_{R}(A) \subseteq$ $\cup_{x \in A} N_{R}(x), \quad \operatorname{cl}_{R}(B) \subseteq \cup_{y \in B} N_{R}(y), \quad$ and $\cup_{x \in A} N_{R}(x) \cap$ $\cup_{y \in B} N_{R}(y)=\varnothing$.

Theorem 2. For any binary relation $R$, the closure space $\left(X, c l_{R}\right)$ is normal if and only iffor every closed set $c l_{R}(A)=A$ contained in $\cup_{x \in A} N_{R}(x)$, there exists some $y$ such that $c l_{R}(A) \subseteq U_{y \in A} N_{R}(y) \subseteq c l_{R}\left(U_{y \in A} N_{R}(y)\right) \subseteq \cup_{x \in A} N_{R}(x)$.

Proof. Let $\left(X, \mathrm{cl}_{R}\right)$ be a normal closure space and $\mathrm{cl}_{R}(A)=$ $A$ be a closed set contained in $\cup_{x \in A} N_{R}(x)$. Thus, $X-\cup_{x \in A} N_{R}(x)=\mathrm{cl}_{R}(B)$ is a closed set which is disjoint from the closed set $\mathrm{cl}_{R}(A)$. Since $\left(X, \mathrm{cl}_{R}\right)$ is normal, there exist $y$ and $z$ with $\cup_{y \in A} N_{R}(y) \cap \cup_{z \in B} N_{R}(z)=\varnothing$ such that $\mathrm{cl}_{R}(A) \subseteq \cup_{y \in A} N_{R}(y)$ and $\mathrm{cl}_{R}(B) \subseteq \cup_{z \in B} N_{R}(z)$ imply $\cup_{y \in A}$ $N_{R}(y) \subseteq\left(X-\cup_{z \in B} N_{R}(z)\right)$. Therefore, $\mathrm{cl}_{R}(A) \subseteq \cup \cup_{y \in A} N_{R}$ $(y) \subseteq X-\cup_{z \in B} N_{R}(z) \subseteq \cup_{x \in A} N_{R}(x)$ Since $\mathrm{cl}_{R}\left(\cup_{y \in A} N_{R}(y)\right)$ is the smallest closed set by Theorem 1, $\mathrm{cl}_{R}(A) \subseteq \quad \cup_{y \in A} N_{R}(y) \subseteq \mathrm{cl}_{R}\left(\cup_{y \in A} N_{R}(y)\right) \subseteq \cup_{x \in A} N_{R}(x)$. Conversely, let $\mathrm{cl}_{R}(A)=A$ and $\mathrm{cl}_{R}(B)=B$ be two closed sets and $\mathrm{cl}_{R}(A)$ be contained in $X-\mathrm{cl}_{R}(B)=\cup_{x \in(X-B)} N_{R}(x)$. By a given condition, there exists some $y$ such that $\mathrm{cl}_{R}(A) \subseteq$ $\cup{ }_{y \in A} N_{R}(y) \subseteq \mathrm{cl}_{R}\left(\cup_{y \in A} N_{R}(y)\right) \subseteq \cup_{x \in A} N_{R}(x)$. Therefore, $\mathrm{cl}_{R}(A) \subseteq \cup_{y \in A} N_{R}(y), \mathrm{cl}_{R}(B) \subseteq X-\mathrm{cl}_{R}\left(\cup_{y \in A} N_{R}(y)\right)$, and $\cup_{y \in A} \quad N_{R}(y) \cap\left(X-\mathrm{cl}_{R}\left(\cup_{y \in A} N_{R}(y)\right)\right)=\varnothing$. Hence, $\left(X, \mathrm{cl}_{R}\right)$ is normal.

Theorem 3. In a normal closure space $\left(X, c l_{R}\right)$, for every pair of disjoint closed sets $c l_{R}(A)=A$ and $c l_{R}(B)=B$, there exist $\cup{ }_{y \in A} N_{R}(y)$ and $\cup_{z \in B} N_{R}(z)$ containing $c l_{R}(A)$ and $c l_{R}(B)$ such that $c l_{R}\left(\cup_{y \in A} N_{R}(y)\right) \cap c l_{R}\left(\cup_{z \in B} N_{R}(z)\right)=\varnothing$. 


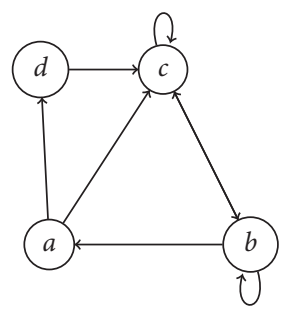

FIgURE 1: Closure space generated from this graph is not normal.

Proof. Let $\left(X, \mathrm{cl}_{R}\right)$ be a normal closure space and $\mathrm{cl}_{R}(A)=$ $A$ and $\operatorname{cl}_{R}(B)=B$ be two disjoint closed sets in $X$. Since $\mathrm{cl}_{R}(A) \cap \mathrm{cl}_{R}(B)=\varnothing$, we have $\mathrm{cl}_{R}(A) \subseteq\left(X-\mathrm{cl}_{R}(B)\right)$, where $X-\mathrm{cl}_{R}(B)=\cup_{x \in X-B} N_{R}(x)$. By the normal closure space of $X$, there exists some $y$ such that $\operatorname{cl}_{R}(A) \subseteq \cup{ }_{y \in A} N_{R}(y)$ $\subseteq \mathrm{cl}_{R}\left(\cup_{y \in A} N_{R}(y)\right) \subseteq\left(X-\mathrm{cl}_{R}(B)\right)$. Thus, $\mathrm{cl}_{R}\left(\cup_{y \in A} N_{R}(y)\right)$ $\cap \operatorname{cl}_{R}(B)=\varnothing$. Since $\operatorname{cl}_{R}(B) \subseteq\left(X-\operatorname{cl}_{R}\left(\cup_{y \in A} N_{R}(y)\right)\right)$ $=\cup_{u \in X-c_{R}\left(\cup N_{R}(y)\right)} N_{R}(y)$, so again by using characterization of a normal closure space, there exists some $z$ such that $\quad \mathrm{cl}_{R}(B) \subseteq \cup_{z \in B} N_{R}(z) \subseteq \mathrm{cl}_{R}\left(\cup_{z \in B} N_{R}(z)\right) \subseteq\left(X-\mathrm{cl}_{R}\right.$ $\left.\left(\cup_{y \in A} N_{R}(y)\right)\right)$ which implies $\operatorname{cl}_{R}\left(\cup_{y \in A} N_{R}(y)\right) \cap \mathrm{cl}_{R}$ $\left(\cup_{z \in B} N_{R}(z)\right)=\varnothing$.

In general, normality does not imply $T_{2}$ which is evident from Example 1. For two distinct points ' $c$ ' and ' $d$ ' which are in the form of straight lines, there does not exist disjoint $\langle x\rangle R$ and $\langle y\rangle R$ containing ' $c$ ' and ' $d$,' respectively. But in $T_{1}$ closure space, the result is true, as shown in Theorem 4 below.

Theorem 4. In a reflexive $T_{1}$ closure space $\left(X, c l_{R}\right)$, every normal space is $T_{2}$.

Proof. Let $\left(X, \mathrm{cl}_{R}\right)$ be a $T_{1}$ normal closure space and $x$ and $y$ be two distinct points. We have to show that $\left(X, \mathrm{cl}_{R}\right)$ is $T_{2}$. Since $\{x\}$ and $\{y\}$ are closed, by normality of $\left(X, \mathrm{cl}_{R}\right)$, there exist some $x$ and $y$ such that $\{x\} \subseteq \cup N_{R}(x),\{y\} \subseteq \cup N_{R}(y)$, and $\cup N_{R}(x) \cap \cup N_{R}(y)=\varnothing$. Hence, $\left(X, \mathrm{cl}_{R}\right)$ is $T_{2}$.

Definition 8. A closure space $\left(X, \mathrm{cl}_{R}\right)$ generated from a binary relation $R$ is said to be almost normal if for a closed set $\mathrm{cl}_{R}(A)=A$ and a regularly closed set $\mathrm{cl}_{R}\left(\operatorname{int}_{R}(B)\right)=B$ disjoint from $\mathrm{cl}_{R}(A)$, there exist some $x$ and $y$, respectively, such that $\operatorname{cl}_{R}(A) \subseteq \cup_{x \in A} N_{R}(x)=U, \quad \mathrm{cl}_{R}\left(\operatorname{int}_{R}(B)\right) \subseteq$ $\cup_{y \in B} N_{R}(y)=V$, and $U \cap V=\varnothing$.

From the definitions, it is obvious that every normal closure space is almost normal but the converse need not be true, as shown in the following example.

Example 3. Let us consider the directed graph in Figure 2. It is clear that $a R=\{b, c, d\}, b R=\{a, c\}, c R=\varnothing$, and $d R=\{a, c\}$. Then, $\langle a\rangle R=\{a, c\},\langle b\rangle R=\{b, c, d\},\langle c\rangle R=\{c\}$, and $\langle d\rangle R=\{b, c, d\}$. The closure space $\left(X, \mathrm{cl}_{R}\right)$ generated from the graph in Figure 2 is almost normal but not normal because for two disjoint closed sets $\mathrm{cl}_{R}(A)=\{a\}=A$ and $\mathrm{cl}_{R}(B)=\{b, d\}=B$, there does not exist some $x$ and $y$ such that $\operatorname{cl}_{R}(A) \subseteq \cup_{x \in A} N_{R}(x), \quad \mathrm{cl}_{R}(B) \subseteq \cup_{y \in B} N_{R}(y), \quad$ and $\cup_{x \in A} N_{R}(x) \cap \cup_{y \in B} N_{R}(y)=\varnothing$.
Example 4. A closure space generated from a graph which is not almost normal. The closure space $\left(X, \mathrm{cl}_{R}\right)$ generated from the binary relation in Figure 3 is not almost normal because for regularly closed set $\mathrm{cl}_{R}\left(\operatorname{int}_{R}(\{a, b\})\right)=\{a, b\}$ and a closed set $\mathrm{cl}_{R}(\{d\})=\{d\}$, there does not exist some $x$ and $y$ such that $\operatorname{cl}_{R}(A) \subseteq \cup_{x \in A} N_{R}(x), \mathrm{cl}_{R}(B) \subseteq \cup_{y \in B} N_{R}(y)$, and $\cup_{x \in A} N_{R}(x) \cap \cup_{y \in B} N_{R}(y)=\varnothing$.

The implications in Figure 4 are obvious from the definitions. But none of these implications is reversible (see [10] and Examples 1, 3, and 4 above).

Theorem 5. A closure space $\left(X, c l_{R}\right)$ generated from a binary relation $R$ is almost normal if and only if for every regularly closed set $c_{R}\left(\operatorname{int}_{R}(A)\right)=A$ contained in $\cup_{x \in A}\left(N_{R}(x)\right)$, there exists some $y$ such that $c_{R}\left(\operatorname{int}_{R}(A)\right) \subseteq \cup, \cup_{y \in A}$ $N_{R}(y) \subseteq c l_{R}\left(\cup_{y \in A} N_{R}(y)\right) \subseteq \cup_{x \in A} N_{R}(x)$.

Proof. Let $\left(X, \mathrm{cl}_{R}\right)$ be an almost normal closure space, $\mathrm{cl}_{R}\left(\operatorname{int}_{R}(A)\right)=A$ be a regularly closed set contained in $\cup_{x \in A} N_{R}(x)$, and $X-\left(\cup_{x \in A}\left(N_{R}(x)\right)\right)=\mathrm{cl}_{R}(B)=B$ be a closed set disjoint from $\operatorname{cl}_{R}\left(\operatorname{int}_{R}(A)\right)=A$. Since $\left(X, \mathrm{cl}_{R}\right)$ is almost normal, there exist some $y$ and $z$ with $\cup_{y \in A}$ $N_{R}(y) \cap \cup_{z \in B} N_{R}(z)=\varnothing$ such that $\operatorname{cl}_{R}\left(\operatorname{int}_{R}(A)\right) \subseteq \cup \cup_{y \in A}$ $N_{R}(y)$ and $\operatorname{cl}_{R}(B) \subseteq \cup_{z \in B} N_{R}(z)$ which imply that $\cup_{y \in A}$ $N_{R}(y) \subseteq X-\cup_{z \in B} N_{R}(z)$. Therefore, $\operatorname{cl}_{R}\left(\right.$ int $\left._{R}(A)\right) \subseteq \cup_{y \in A}$ $N_{R}(y) \subseteq\left(X-\cup_{z \in B} N_{R}(z)\right) \subseteq \cup_{x \in A} N_{R}(x)$. Since $c_{R}\left(\cup_{y \in A}\right.$ $\left.N_{R}(y)\right)$ is the smallest closed set by Theorem $1, \mathrm{cl}_{R}$ $\left(\operatorname{int}_{R}(A)\right) \subseteq \cup_{y \in A} N_{R}(y) \subseteq \operatorname{cl}_{R}\left(\cup_{y \in A} N_{R}(y)\right) \subseteq \cup_{x \in A} N_{R}$ $(x)$. Conversely, let $\mathrm{cl}_{R}\left(\operatorname{int}_{R}(A)\right)=A$ be a regularly closed set and $\operatorname{cl}_{R}(B)=B$ be a closed set. Thus, $X-\mathrm{cl}_{R}$ $(B)=\cup_{x \in(X-B)} N_{R}(x)$ is an open set containing $\mathrm{cl}_{R}\left(\right.$ int $_{R}$ $(A))$. By the given condition, there exists some $y$ such that $\mathrm{cl}_{R}\left(\operatorname{int}_{R}(A)\right) \subseteq \cup_{y \in A} N_{R}(y) \subseteq \mathrm{cl}_{R}\left(\cup_{y \in A} N_{R}(y)\right) \subseteq \cup_{x \in(X-B)}$ $N_{R}(x)$. Thus, $\operatorname{cl}_{R}\left(\operatorname{int}_{R}(A)\right) \subseteq \cup_{y \in A} N_{R}(y)$ and $\operatorname{cl}_{R}(B)$ $\subseteq X-\mathrm{cl}_{R}\left(\cup_{y \in A} N_{R}(y)\right)$. Hence, $\left(X, \mathrm{cl}_{R}\right)$ is almost normal.

Theorem 6. If $R$ a binary relation, then the generated closure space $\left(X, c l_{R}\right)$ is almost normal if and only if for every closed set $c l_{R}(A)=A$ contained in a regularly open set $\cup_{x \in A} N_{R}(x)$, there exists some $y$ such that $c_{R}(A) \subseteq \cup_{x \in A} N_{R}(y)$ $\subseteq c l_{R}\left(\cup_{x \in A} N_{R}(y)\right) \subseteq \cup_{x \in A} N_{R}(x)$.

Proof. Let $\left(X, \mathrm{cl}_{R}\right)$ be an almost normal closure space, $\mathrm{cl}_{R}(A)=A$ be a closed set contained in $\cup_{x \in A} N_{R}(x)$, and $X-\left(\cup_{x \in A} N_{R}(x)\right)=\mathrm{cl}_{R}\left(\operatorname{int}_{R}(B)\right)=B$ be a regularly closed set disjoint from $\mathrm{cl}_{R}(A)$. Since $\left(X, \mathrm{cl}_{R}\right)$ is an almost normal closure space, there exist some $y$ and $z$ with $\cup_{y \in A}$ $N_{R}(y) \cap \cup_{z \in B} N_{R}(z)=\varnothing$ such that $\operatorname{cl}_{R}(A) \subseteq \cup{ }_{y \in A} N_{R}(y)$ and $\operatorname{cl}_{R}$ int $_{R}(B) \subseteq \cup_{z \in B} N_{R}(z)$ which imply that $\cup_{y \in A} N_{R}(y) \subseteq X-\cup_{z \in B} N_{R}(z)$. Therefore, $\operatorname{cl}_{R}(A) \subseteq \cup \quad \cup_{y \in A}$ $N_{R}(y) \subseteq X-\cup_{z \in B} N_{R}(z) \subseteq \cup_{x \in A} N_{R}(x)$ Since $\mathrm{cl}_{R}\left(\cup_{y \in A} N_{R}(y)\right)$ is the smallest closed set by Theorem 1, $\mathrm{cl}_{R}(A) \subseteq \cup_{y \in A} N_{R}(y) \subseteq \mathrm{cl}_{R}\left(\cup_{y \in A} N_{R}(y)\right) \subseteq \cup_{x \in A} N_{R}(x)$.

Conversely, let $\mathrm{cl}_{R}(A)=A$ be a closed set and $\mathrm{cl}_{R}\left(\right.$ int $\left._{R}(B)\right)=B$ be a regularly closed sets' disjoint from a closed set. Thus, $X-\mathrm{cl}_{R}\left(\operatorname{int}_{R}(B)\right)=\cup_{x \in(X-B)} N_{R}(x)$ is a regularly open set containing $\mathrm{cl}_{R}(A)$. By the given condition, there exists some $y$ such that 


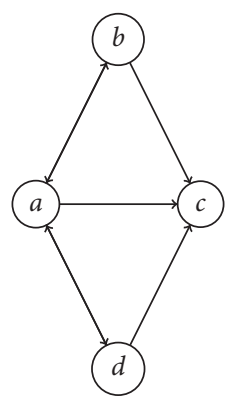

Figure 2: Closure space generated from this graph is almost normal but not normal.

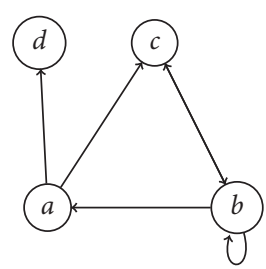

FIgURE 3: Closure space generated from this graph is not almost normal.

Strongly normal $\longrightarrow$ Normal $\longrightarrow$ Almost normal

Figure 4: Interrelation diagram.

$\mathrm{cl}_{R}(A) \subseteq \cup_{y \in A} N_{R}(y) \subseteq \mathrm{cl}_{R}\left(\cup_{y \in A} N_{R}(y)\right) \subseteq$ $\cup_{x \in(X-B)} N_{R}(x)$. Thus, $\mathrm{cl}_{R}(A) \subseteq \cup_{y \in A} N_{R}(y)$ and $\mathrm{cl}_{R}\left(\right.$ int $_{R}$ $(B)) \subseteq X-\mathrm{cl}_{R}\left(\cup_{y \in A} N_{R}(y)\right)$. Hence, $\left(X, \mathrm{cl}_{R}\right)$ is almost normal.

Definition 9. Let $R$ be any binary relation on $X$ and $\left(X, \mathrm{cl}_{R}\right)$ be the generated closure space. Then, a family $\mathscr{W}=\left\{W_{\alpha}: \alpha \in \Lambda\right\}$ of subset $X$ is said to be an $R$-cover of $X$ if $X=\cup_{W_{\alpha} \in \mathscr{W}} W_{\alpha}$. If elements of $\mathscr{W}$ are of the form $\cup N_{R}(x)$, then it is said to be an $R$-open cover of $X$.

Definition 10. A closure space $\left(X, \mathrm{cl}_{R}\right)$ is said to be nearly compact if for every $R$-open cover $\left\{W_{\alpha}: \alpha \in \Lambda\right\}$ of $X$, there is a finite subset $P$ of $\Lambda$ such that $\left\{\operatorname{int}\left(\operatorname{cl}_{R}\left(W_{\alpha}\right)\right): \alpha \in P\right\}$ is an $R$-cover of $X$.

Theorem 7. Every regularly closed closure subspace of a nearly compact space is nearly compact.

Proof. Let $\left(Y, \mathrm{cl}_{R_{\mathrm{Y}}}\right)$ be a regularly closed closure subspace of a nearly compact space $\left(X, \mathrm{cl}_{R}\right)$. Let $\mathscr{U}=\cup\left\{\cup N_{R}\right.$ $\left.\left(x_{\alpha}\right): \alpha \in Q\right\}$ be an $R$-open cover of $Y$. We have to show that there is a finite subcollection whose closure is an $R$-cover of $Y$. Since $\left\{N_{R}\left(x_{\alpha}\right): \alpha \in Q\right\}$ is an $R$-open cover of $Y, \cup\left\{\cup N_{R}\right.$ $\left.\left(x_{\alpha}\right): \alpha \in Q\right\} \cup(X-Y)$ is an $R$-open cover of $X$. Also, $\left(X, \mathrm{cl}_{R}\right)$ is nearly compact, so there is a finite subcollection $\left\{N_{R}\left(x_{\alpha}\right): \alpha \in P\right\}$ such that $\left\{\operatorname{int}\left(\mathrm{cl}_{R}\left(N_{R}\left(x_{\alpha}\right)\right)\right): \alpha \in P\right\}$ is an $R$-cover of $X$. If this subcollection has $(X-Y)$, then discard it. The remaining subcollection also covers $Y$. Hence, $\left(Y, \mathrm{cl}_{R}\right)$ is a nearly compact closure space.

Theorem 8. The closure space $\left(X, l_{R}\right)$ generated from a reflexive relation $R$ is almost normal if it is nearly compact and $T_{2}$.

Proof. Let $\mathrm{cl}_{R}(A)=A$ be a regularly closed set which is disjoint from a closed set $\mathrm{cl}_{R}(B)=B$. We have to show that $\left(X, \mathrm{cl}_{R}\right)$ is almost normal. Since for a point $b \in \mathrm{cl}_{R}(B)$ and a point $a \in \mathrm{cl}_{R}(A), \quad\langle a\rangle R \cap\langle b\rangle R=\varnothing$. The family $\mathcal{U}=\cup\left\{\cup N_{R}(a): a \in \operatorname{cl}_{R}(A)\right\}$ covers $A$. Since $\operatorname{cl}_{R}(A)$ is regularly closed and $\left(X, \mathrm{cl}_{R}\right)$ is nearly compact, $\operatorname{cl}_{R}(A)$ is nearly compact by Theorem 7. Thus, there is a finite set $Q$ of $\operatorname{cl}_{R}(A)$ such that $\left\{\operatorname{int}_{R}\left(\operatorname{cl}_{R}\left(\cup N_{R}\left(a_{\alpha}\right)\right)\right): \alpha \in Q\right\}$ is an $R$ cover of $\operatorname{cl}_{R}(A)$. Thus, there exist some $a$ and $b$ such that $c l_{R}(A) \subseteq \cup\left(\operatorname{int}_{R}\left(\mathrm{cl}_{R}\left(\cup N_{R}\left(a_{\alpha}\right)\right)\right)\right), b \in N_{R}(b)$, and $\cup N_{R}$ $\left(a_{\alpha}\right) \cap N_{R}(b)=\varnothing$. Since for every point $b \in \mathrm{cl}_{R}(B)$ there exists $N_{R}(b)$, the collection $\cup\left\{\cup N_{R}\left(b_{\alpha}\right): \alpha \in \mathrm{cl}_{R}(b)\right\}$ covers $\operatorname{cl}_{R}(B)$. Thus, $\operatorname{cl}_{R}(A) \subseteq \operatorname{int}_{R}\left(\operatorname{cl}_{R}\left(\cup_{a \in A} N_{R}\left(a_{\alpha}\right)\right)\right)$ and $\mathrm{cl}_{R}(B) \subseteq \operatorname{int}_{R}\left(\mathrm{cl}_{R}\left(\mathrm{U}_{b \in B} N_{R}\left(b_{\alpha}\right)\right)\right)$ as $\left(X, \mathrm{cl}_{R}\right)$ is nearly compact and $\cup \operatorname{int}_{R}\left(\operatorname{cl}_{R}\left(\cup_{a \in A} N_{R}\left(a_{\alpha}\right)\right)\right) \cap \operatorname{int}_{R}\left(c_{R}\left(\cup_{b \in B}\right.\right.$ $\left.\left.N_{R}\left(b_{\alpha}\right)\right)\right)=\varnothing$. Hence, $\left(X, \mathrm{cl}_{R}\right)$ is an almost normal closure space.

Remark 2. The following example establishes that $T_{2}$ condition in Theorem 8 cannot be dropped.

Example 5. Let us consider the directed graph in Figure 5. Here, the closure space $\left(X, \mathrm{cl}_{R}\right)$ generated from the graph is nearly compact but not almost normal because for the regularly closed set $\mathrm{cl}_{R}\left(\operatorname{int}_{R}(A)\right)=\{a, b\}$ and a closed set $\mathrm{cl}_{R}(B)=\{d\}$, there does not exist some $x$ and $y$ such that $\mathrm{cl}_{R}\left(\operatorname{int}_{R}(A)\right) \subseteq \mathrm{U}_{x \in A}\left(N_{R}(x)\right), \operatorname{cl}_{R}(B) \subseteq \cup_{x \in y}\left(N_{R}(y)\right)$, and $\cup_{x \in A}\left(N_{R}(x)\right) \cap \cup_{x \in y}\left(N_{R}(y)\right)=\varnothing$.

\section{Subspace}

Definition 11 (see [5]). Let $Y \subseteq X$ and $R_{Y} \subseteq R$; then, $\left(Y, \mathrm{cl}_{R_{Y}}\right)$ is called a closure subspace of a closure space $\left(X, \mathrm{cl}_{R}\right)$ if $\langle x\rangle R_{Y}=\langle x\rangle R \cap Y$ for all $x \in Y$.

Remark 3 (see [12]). Let $Y$ be a subspace of a closure space $X$. Then,

(a) $A$ is closed (open) in $X$ implies that $Y \cap A$ is closed (open) in $Y$.

(b) $Y$ is closed in $X$ and $A$ is a closed set in $Y$ imply that $A$ is closed in $X$.

In the following example, it is shown that the normality of the closure space generated from a graph does not imply that the closure space generated from its subgraph is normal.

Example 6. Let us consider the graph in Figure 6. The closure space $\left(X, \mathrm{cl}_{R_{X}}\right)$ generated from the graph in Figure 6 is normal as it satisfies the condition of a normal closure space. But the closure space generated from its subgraph, as shown in Figure 7, is not normal. 


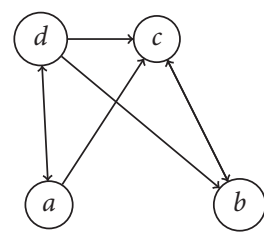

FIgURe 5: Closure space generated from this relation is nearly compact but not almost normal.

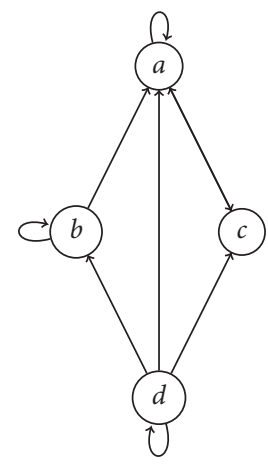

FIGURE 6: Closure space generated from this graph is normal.

The closure space $\left(Y, \mathrm{cl}_{R_{Y}}\right)$ of the subgraph in Figure 7 is not normal because for two disjoint closed sets $\mathrm{cl}_{R_{Y}}(A)=$ $\{b\}=A$ and $\mathrm{cl}_{R_{Y}}(B)=\{c\}=B$, there does not exist some $x$ and $y$ in $Y$ such that $\operatorname{cl}_{R_{Y}}(A) \subseteq \cup_{x \in A} N_{R_{Y}}(x), \operatorname{cl}_{R_{Y}}(B) \subseteq$ $\cup_{y \in B} N_{R_{Y}}(y)$, and $\cup_{x \in A} N_{R_{Y}}(x) \cap \cup_{y \in B} N_{R_{Y}}(y)=\varnothing$.

Theorem 9. Let $\left(X, c l_{R}\right)$ be a normal closure space. Then, every closed subspace $\left(Y, c l_{R_{Y}}\right)$ of $\left(X, c l_{R}\right)$ is normal.

Proof. Let $A=\mathrm{cl}_{R_{Y}}(A)$ and $B=\mathrm{cl}_{R_{Y}}(B)$ be two disjoint closed sets in a closed subspace $Y$ of $X$. By Remark $3, \mathrm{cl}_{R}(A)$ and $\operatorname{cl}_{R}(B)$ closed in $X$ implies that $\mathrm{cl}_{R}(A) \cap Y$ and $\mathrm{cl}_{R}(B) \cap Y$ are closed in $X$. By Remark $3, \mathrm{cl}_{R_{Y}}(A)$ and $\mathrm{cl}_{R_{Y}}(B)$ are closed in $X$. Since $\left(X, \mathrm{cl}_{R}\right)$ is normal, there exist some $x$ and $y$ such that $\operatorname{cl}_{R_{Y}}(A) \subseteq \cup_{x \in A}\left(N_{R}(x)\right), \quad \mathrm{cl}_{R_{Y}}(B) \subseteq$ $\cup_{y \in B}\left(N_{R}(y)\right)$, and $\cup_{x \in A}\left(N_{R}(x)\right) \cap \cup_{y \in B}\left(N_{R}(y)\right)=\varnothing$. Thus, by Definition 11, $\cup_{x \in A}\left(N_{R}(x)\right) \cap Y$ and $U_{y \in B}$ $\left(N_{R}(y)\right) \cap Y$ contain $\mathrm{cl}_{R_{Y}}(A), \mathrm{cl}_{R_{Y}}(B)$, and $\cup_{x \in A}\left(N_{R}(x)\right)$ $\cap Y \cap \cup_{y \in B}\left(N_{R}(y)\right) \cap Y=\varnothing$. Hence, $\left(Y, \mathrm{cl}_{R_{Y}}\right)$ is normal.

Remark 4. A closed subspace of a normal closure space is normal. But a closed subgraph of an almost normal space need not be almost normal, as shown in Example 7 below.

Example 7. Closure space generated from a closed subgraph of an almost normal closure space need not be almost normal.

The closure space $\left(X, \mathrm{cl}_{R_{X}}\right)$ generated from the graph in Figure 8 is almost normal because for every closed set and a regularly closed set disjoint from a closed set, there exist some $x$ and $y$ satisfying the condition of the almost normal closure space.

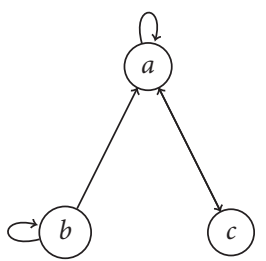

Figure 7: Closure space generated from this subgraph is not normal.

Let us consider the closed subgraph in Figure 9 of the graph in Figure 8. The closed closure subspace $\left(Y, \mathrm{cl}_{R_{Y}}\right)$ generated from the subgraph in Figure 9 is not almost normal because for a closed set $A=\{a\}=\mathrm{cl}_{R_{Y}}(A)$ and a regularly closed set $B=\{d, e\}=\mathrm{cl}_{R_{Y}}\left(\operatorname{int}_{R_{Y}}(B)\right)$, there does not exist some $x$ and $y$ in $Y$ satisfying the required condition.

Remark 5. In a closure space $\left(X, \mathrm{cl}_{R}\right)$, a regularly closed closure subspace of an almost normal closure space need not be almost normal. We can say that a clopen subspace of an almost normal space $\left(X, \mathrm{cl}_{R}\right)$ is almost normal.

\section{Preservation under Mapping}

Definition 12 (see [5]). Let $\left(X_{1}, \mathrm{cl}_{R_{1}}\right)$ and $\left(X_{2}, \mathrm{cl}_{R_{2}}\right)$ be two closure spaces. A function $f: X_{1} \longrightarrow X_{2}$ is continuous at $x \in X_{1}$ if and only if $f\left(\langle x\rangle R_{1}\right) \subseteq\langle f(x)\rangle R_{2}$. A function from a closure space $\left(X_{1}, \mathrm{cl}_{R_{1}}\right)$ into a closure space $\left(X_{2}, \mathrm{cl}_{R_{2}}\right)$ is said to be continuous on $X_{1}$ if and only if it is continuous at each point of $X_{1}$.

Theorem 10 (see [5]). Let $f$ be a function from a closure space $\left(X_{1}, c l_{R_{1}}\right)$ into a closure space $\left(X_{2}, c l_{R_{2}}\right)$; then, the following conditions are equivalent:

(1) $f$ is continuous.

(2) For every subset $A$ of $X_{1}, f\left(c l_{R_{1}}(A)\right) \subseteq c l_{R_{2}}(f(A))$.

(3) The inverse image of every closed subset of $X_{2}$ is a closed subset of $X_{1}$.

(4) The inverse image of every open subset of $X_{2}$ is an open subset of $X_{1}$.

Definition 13 (see [5]). A function $f:\left(X_{1}, \mathrm{cl}_{R_{1}}\right)$ $\longrightarrow\left(X_{2}, \mathrm{cl}_{R_{2}}\right)$ is called open (closed) if the image of an open (closed) subset of $X_{1}$ is an open (closed) subset of $X_{2}$.

Theorem 11. Let $f:\left(X, c l_{R_{X}}\right) \longrightarrow\left(Y, c l_{R_{Y}}\right)$ be a continuous closed and surjection and $\left(X, c l_{R_{X}}\right)$ be normal; then, $\left(Y, c l_{R_{Y}}\right)$ is also a normal closure space.

Proof. Let $\mathrm{cl}_{R_{Y}}(A)=A$ and $\operatorname{cl}_{R_{Y}}(B)=B$ be two disjoint closed sets in $Y$. We have to show that $\left(Y, \mathrm{cl}_{R_{Y}}\right)$ is normal. Since $f$ is continuous, $f^{-1}\left(\mathrm{cl}_{R_{Y}}(A)\right)$ and $f^{-1}\left(\mathrm{cl}_{R_{Y}}(B)\right)$ are disjoint closed sets in $X$. As $\left(X, \mathrm{cl}_{R_{X}}\right)$ is normal, there exist some $x$ and $y$ with $\cup_{x \in A} N_{R_{X}}(x) \cap \cup_{y \in B} N_{R_{X}}(y)=\varnothing$ such that $f^{-1}\left(\mathrm{cl}_{R_{Y}}(A)\right) \subseteq \cup_{x \in A} N_{R_{X}}(x)$ and $f^{-1}\left(\mathrm{cl}_{R_{Y}}(B)\right) \subseteq$ $\cup{ }_{y \in B} N_{R_{X}}(y)$. Since $f$ is closed, $f\left(X-\cup_{x \in A} N_{R_{X}}(x)\right)$ and $f\left(X-\cup_{y \in B} N_{R_{X}}(y)\right)$ are closed in $Y$. Since $f^{-1}\left(\mathrm{cl}_{R_{Y}}\right.$ 


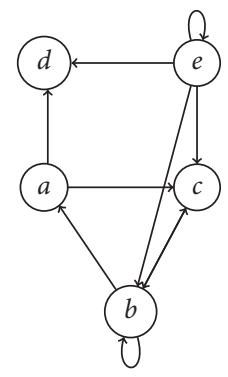

Figure 8: Closure space generated from this graph is almost normal.

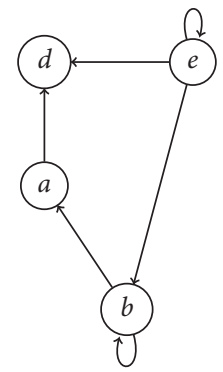

FIGURE 9: Closure space generated from this subgraph is not almost normal.

$(A)) \subseteq \cup_{x \in A} N_{R_{X}}(x), \quad\left(X-\cup_{x \in A} N_{R_{X}}(x)\right) \subseteq\left(X-f^{-1}\left(\mathrm{cl}_{R_{Y}}\right.\right.$ (A)) implies that $f\left(X-\cup_{x \in A} N_{R_{X}}(x)\right) \subseteq f\left(X-f^{-1}\right.$ $\left.\left(\mathrm{cl}_{R_{Y}}(A)\right)\right)$. Thus, $\mathrm{cl}_{R_{Y}}(A) \subseteq\left(Y-f\left(X-\cup_{x \in A} N_{R_{X}}(x)\right)\right)=$ $\cup_{u \in\left(Y-f\left(X-\cup N_{R_{X}}(x)\right)\right)} N_{R_{Y}}(u)$. Since $f^{-1}\left(\cup_{u \in\left(Y-f\left(X-\cup N_{R_{X}}(x)\right)\right)}\right.$ $\left.N_{R_{Y}}(u)\right)=\cup_{x \in A} N_{R_{X}}(x)$, there exists $\cup_{u \in A} N_{R_{Y}}(u){ }^{R_{X}}$ containing $\mathrm{cl}_{R_{Y}}(A)$ such that $f^{-1}\left(\cup_{u \in A} N_{R_{Y}}(u)\right) \subseteq \cup_{x \in A} N_{R_{X}}(x)$. Similarly, there exists $\cup_{v \in B} N_{R_{Y}}(v)$ containing $\mathrm{cl}_{R_{Y}}(B)$ such that $f^{-1}\left(\cup_{v \in B} N_{R_{Y}}(v)\right) \subseteq \cup_{y \in B} N_{R_{X}}(y)$. Also, $f^{-1}\left(\cup_{u \in A} N_{R_{Y}}\right.$ $(u)) \cap f^{-1}\left(\cup_{v \in B} N_{R_{Y}}(v)\right) \subseteq \cup_{x \in A} N_{R_{X}}(x) \cap \cup_{y \in B} N_{R_{X}}(y)=$ $\varnothing$ implies that $\left(\cup_{u \in A} N_{R_{Y}}(u)\right) \cap\left(\cup_{v \in B} N_{R_{Y}}(v)\right)=\varnothing$. Hence, $\left(Y, \mathrm{cl}_{R_{Y}}\right)$ is a normal closure space.

Example 8. In a closure space $\left(X, \mathrm{cl}_{R_{X}}\right)$, the continuous image of an almost normal space need not be almost normal.

Let $X$ be a set of natural numbers and $Y=\{a, b, c, d\}$ be a set. Let $R_{1}$ be a relation defined on $X$ as $a R_{1} b$ if and only if $a=b$ for all $a$ and $b \in X$ and $b=a-1$, where $a$ is even, and $R_{2}=\{(a, c),(a, d),(b, a),(b, b),(b, c),(c, b)\}$ be a relation defined on $Y$. Then,

$$
\langle i\rangle R_{1}= \begin{cases}i, & \text { if } i \text { is odd, } \\ i-1, i, & \text { if } i \text { is even }\end{cases}
$$

and $\langle x\rangle R_{2}$ is defined as in Example 4. Here, $\left(X, \mathrm{cl}_{R_{1}}\right)$ and $\left(Y, \mathrm{cl}_{R_{2}}\right)$ are closure spaces. A function $f:\left(X, \mathrm{cl}_{R_{1}}\right)$ $\longrightarrow\left(Y, \mathrm{cl}_{R_{2}}\right)$,

$$
f(x)= \begin{cases}a, & \text { if } x \in \mathbb{N}-\{1,3,4\}, \\ b, & \text { if } x=1 \\ c, & \text { if } x=3 \\ d, & \text { if } x=4\end{cases}
$$

is continuous. Here, closure space $\left(X, \mathrm{cl}_{R_{1}}\right)$ is almost normal but $\left(Y, \mathrm{cl}_{R_{2}}\right)$ is not almost normal because for closed set $\mathrm{cl}_{R_{2}}(\{d\})=\{d\}=A$ and a regularly closed set $\mathrm{cl}_{R_{2}}\left(\right.$ int $\left._{R_{2}}(B)\right)=\{a, b\}=B$, there does not exist some $x$ and $y$ in $Y$ such that $\operatorname{cl}_{R_{2}}(\{d\}) \subseteq\left(\cup_{x \in A} N_{R_{2}}(x)\right), \mathrm{cl}_{R_{2}}\left(\operatorname{int}_{R_{2}}(\{a, b\})\right)$ $\subseteq\left(\cup_{y \in B} N_{R_{2}}(y)\right)$, and $\cup_{x \in A} N_{R_{2}}(x)^{2} \cap \cup_{y \in B} N_{R_{2}}(y)=\varnothing$.

Example 9. continuous closed image of an almost normal space need not be almost normal.

Let $X=\mathbb{N} \cup\{p, q\}$ and $Y=\{a, b, c, d\}$ be two sets. Let $R_{1}$ be a relation defined on $X$ as $a R_{1} b$ if and only if

$$
\begin{array}{ll}
a=b, & \forall a \text { and } b \in X, \\
b=p, & \text { for } a \geq 4, \\
b=a-1, & \text { for } a=3, \\
b=a+1, & \text { for } a=2, \\
b=1, & \text { for } a=q,
\end{array}
$$

and relation $R_{2}=\{(a, a),(a, b),(a, c),(b, c),(c, b),(d, c)$, $(d, d)\}$, defined on $Y$, are two binary relations. Then, $\langle 1\rangle R_{1}=\{1\},\langle 2\rangle R_{1}=\langle 3\rangle R_{1}=\{2,3\},\langle i\rangle R_{1}=\{i, p\}$ for $i \geq 4$, $\langle p\rangle R_{1}=\{p\},\langle q\rangle R_{1}=\{1, q\}, \quad\langle a\rangle R_{2}=\{a, b, c\}, \quad\langle b\rangle R_{2}=$ $\{b\},\langle c\rangle R_{2}=\{c\}$, and $\langle d\rangle R_{2}=\{c, d\}$. Here, $\left(X, \mathrm{cl}_{R_{1}}\right)$ and $\left(Y, \mathrm{cl}_{R_{2}}\right)$ are closure spaces. A function $f:\left(X, \mathrm{cl}_{R_{1}}\right) \longrightarrow$ $\left(Y, \mathrm{cl}_{R_{2}}\right)$ is defined as

$$
f(x)= \begin{cases}a, & \text { if } x=q, 2,3, \text { and } 4, \\ b, & \text { if } x=1, \\ c, & \text { if } x=p \\ d, & \text { if } x \in \mathbb{N}-\{1,2,3,4\}\end{cases}
$$

is continuous. Here, $\left(X, \mathrm{cl}_{R_{1}}\right)$ is almost normal but $\left(Y, \mathrm{cl}_{R_{2}}\right)$ is not almost normal because for closed set $\mathrm{cl}_{R_{2}}(\{d\})=\{d\}=$ $A$ and a regularly closed set $\mathrm{cl}_{R_{2}}\left(\operatorname{int}_{R_{2}}(\{a, b\})\right)=\{a, b\}=B$, there does not exist some $x$ and $y$ in $Y$ satisfying the condition of the almost normal closure space.

Since the continuous closed image of an almost normal space need not be almost normal, so we replace a continuous function with a completely continuous.

Definition 14. Let $R$ be a binary relation and $\left(X, \mathrm{cl}_{R_{X}}\right)$ and $\left(Y, \mathrm{cl}_{R_{Y}}\right)$ be two closure spaces; then, a function $f:\left(X, \mathrm{cl}_{R_{X}}\right) \longrightarrow\left(Y, \mathrm{cl}_{R_{Y}}\right)$ is said to be completely continuous if inverse image of $\bigcup N_{R}(x)=U$ in $\left(Y, \mathrm{cl}_{R_{Y}}\right)$ is regularly open in $\left(X, \mathrm{cl}_{R_{X}}\right)$.

From the above definition, we can say that if $f:\left(X, \mathrm{cl}_{R_{X}}\right) \longrightarrow\left(Y, \mathrm{cl}_{R_{Y}}\right)$ is a completely continuous onto function and $B=\mathrm{cl}_{R_{Y}}\left(\operatorname{int}_{R_{Y}}(B)\right)$ is regularly closed in $\left(Y, \mathrm{cl}_{R_{Y}}\right)$, then $f^{-1}(B)$ is regularly closed in $\left(X, \mathrm{cl}_{R_{X}}\right)$.

Observation 1 . In a closure space $\left(X, \mathrm{cl}_{R_{X}}\right)$, every completely continuous function is continuous.

Example 10. The completely continuous image of an almost normal space in a closure space need not be almost normal. 
Let $X$ be a set of natural numbers and $Y=\{p, q, r, s\}$ be two sets. Let $R_{1}$ be a relation on $X$, as defined in Example 8, and $R_{2}$ be the relation on $Y$ given by $R_{2}=\{(p, p),(q, p)$, $(q, q),(q, r),(r, r),(s, r),(s, s)\}$. Then, $\langle i\rangle R_{1}$ is defined as in Example 8, and $\langle p\rangle R_{2}=\{p\},\langle q\rangle R_{2}=\{p, q, r\},\langle r\rangle R_{2}=\{r\}$, and $\langle s\rangle R_{2}=\{r, s\}$. Here, $\left(X, \mathrm{cl}_{R_{1}}\right)$ and $\left(Y, \mathrm{cl}_{R_{2}}\right)$ are closure spaces. A function $f:\left(X, \mathrm{cl}_{R_{1}}\right) \longrightarrow\left(Y, \mathrm{cl}_{R_{2}}\right)$ defined by

$$
f(x)= \begin{cases}p, & \text { if } x=1 \text { and } 2, \\ q, & \text { if } x=\mathbb{N}-\{1,2,3,4,5,6\}, \\ r, & \text { if } x=3 \text { and } 4, \\ s, & \text { if } x=5 \text { and } 6,\end{cases}
$$

is completely continuous. Here, $\left(X, \mathrm{cl}_{R_{1}}\right)$ is almost normal but $\left(Y, \mathrm{cl}_{R_{2}}\right)$ is not almost normal because for a closed set $A=\{s\}=\mathrm{cl}_{R_{2}}(A)$ and a regularly closed set $B=\{p, q\}=\mathrm{cl}_{R_{2}}\left(\operatorname{int}_{R_{2}}(B)\right)$, there does not exist some $x$ and $y$ in $Y$ such that $\operatorname{cl}_{R_{2}}(A) \subseteq\left(\cup_{x \in A} N_{R_{2}}(x)\right), \operatorname{cl}_{R_{2}}\left(\right.$ int $_{R_{2}}$ $(B)) \subseteq\left(\cup_{y \in B} N_{R_{2}}(y)\right)$, $\cup_{x \in A} N_{R_{2}}(x) \cap \cup_{y \in B} N_{R_{2}}(y)=\varnothing$.

Theorem 12. Completely continuous, closed image of an almost normal closure space, is almost normal.

Proof. Let cl $\mathrm{R}_{R_{Y}}(A)=A$ be a closed set and $\mathrm{cl}_{R_{Y}}\left(\operatorname{int}_{R_{Y}}(B)\right)=$ $B$ be a regularly closed set. Then, $f^{-1}\left(\mathrm{cl}_{R_{Y}}(A)\right)$ is closed, and $f^{-1}\left(c_{R_{Y}}\left(\operatorname{int}_{R_{Y}}(B)\right)\right)$ is regularly closed in $X$. Since $\left(X, \mathrm{cl}_{R_{X}}\right)$ is almost normal, there exist some $x$ and $y$ with $\cup_{x \in A}$ $\left(N_{R_{X}}(x)\right) \cap \cup_{y \in B}\left(N_{R_{X}}(y)\right)=\varnothing$ such that $f^{-1} \mathrm{cl}_{R_{X}}(A) \subseteq$ $\cup_{x \in A}\left(N_{R_{X}}(x)\right)$ and $f^{-1}\left(\operatorname{cl}_{R_{Y}}\left(\operatorname{int}_{R_{Y}}(B)\right)\right) \subseteq \cup_{y \in B}\left(N_{R_{X}}(y)\right)$. Thus, $f\left(X-\cup_{x \in A}\left(N_{R_{X}}(x)\right)\right)$ and $f\left(X-\cup_{y \in B}\left(N_{R_{X}}(y)\right)\right)$ are closed in $\left(Y, \mathrm{cl}_{R_{Y}}\right)$. As $f^{-1}\left(\mathrm{cl}_{R_{Y}}(A)\right) \subseteq \cup_{X \in A}\left(N_{R_{X}}(x)\right)$ implies $X-\cup_{x \in A}\left(N_{R_{X}}(x)\right) \subseteq X-f^{-1}\left(\mathrm{cl}_{R_{Y}}(A)\right), \mathrm{cl}_{R_{Y}}(A) \subseteq$ $\left(Y-f\left(X-\cup_{x \in A}\left(N_{R_{X}}(x)\right)\right)\right)=\cup_{p \in Y-f\left(X-\cup N_{R_{X}}(x)\right)}\left(N_{R_{X}}\right.$ $(p))$. Now, $f^{-1}\left(\cup_{p \in Y-f\left(X-\cup N_{R_{X}}(x)\right)}\left(N_{R_{X}}(p)\right)\right)=f^{-1}(Y-f$ $\left.\left(X-\cup_{x \in A}\left(N_{R_{X}}(x)\right)\right)\right)=\cup_{x \in A}\left(N_{R_{Y}}(x)\right)$. Thus, there exists some $p$ in $Y$ such that $\mathrm{cl}_{R_{Y}}(A) \subseteq \cup_{p \in A}\left(N_{R_{X}}(p)\right)$. Similarly, there exists some $q$ in $Y$ such that $\mathrm{cl}_{R_{Y}}\left(\operatorname{int}_{R_{Y}}(B)\right)$ $\subseteq \cup_{q \in B}\left(N_{R_{X}}(q)\right)$. Also, $\cup_{p \in A}\left(N_{R_{X}}(p)\right) \cup \cup_{q \in B}\left(N_{R_{X}}(q)\right)=$ $\varnothing$. Hence, $\left(X, \mathrm{cl}_{R_{Y}}\right)$ is an almost normal closure space.

\section{Data Availability}

No data were used to support the findings of this study.

\section{Conflicts of Interest}

The authors declare that they have no conflicts of interest.

\section{Acknowledgments}

The first author is thankful to Department of Science and Technology (DST), Government of India, for awarding INSPIRE fellowship (IF140967).

\section{References}

[1] A. Rosenfeld, "Digital topology," Amerian Mathematical Monthly, vol. 86, no. 9, pp. 621-630, 1979.
[2] M. B. Smyth, "Semi-metrics, closure spaces and digital topology," Theoretical Computer Science, vol. 151, no. 1, pp. 257-276, 1995.

[3] J. Šlapal, "Closure operations for digital topology," Theoretical Computer Science, vol. 305, pp. 457-471, 2003.

[4] J. Šlapal, "Graphs with a path partition for structuring digital spaces," Information Sciences, vol. 233, pp. 305-312, 2013.

[5] A. A. Allam, M. Y. Bakeir, and E. A. Abo-Tabl, "New approach for closure spaces by relations," Acta Mathematica Academiae Paedagogicae Nyregyhziensis, vol. 22, pp. 285-304, 2006.

[6] A. A. Allam, M. Y. Bakeir, and E. A. Abo-Tabl, "Some methods for generating topologies by relations," Bulletin of the Malaysian Mathematical Sciences Society, vol. 31, pp. 1-11, 2008.

[7] J. Šlapal and J. L. Pfaltz, "Closure operators associated with networks," Hacettepe Journal of Mathematics and Statistics, vol. 46, no. 1, pp. 91-101, 2017.

[8] B. M. R. Stadler and P. F. Stadler, "Higher separation axioms in generalized closure spaces," Commentationes Mathematicae, vol. 43, no. 2, pp. 257-273, 2003.

[9] R. Gupta and A. K. Das, "Some variants of normal cech closure spaces via canonically closed sets," Mathematics, vol. 9, no. 11, p. 1225, 2021.

[10] R. Gupta and A. K. Das, "New separation axioms on closure spaces generated by relations," Proceedings of the Jangjeon Mathematical Society, vol. 21, no. 1, pp. 23-31, 2018.

[11] G. Liu, "Closures and topological closures in quasi-discrete closure spaces," Applied Mathematics Letters, vol. 23, no. 7, pp. 772-776, 2010.

[12] E. Čech, Topological Spaces, Academia, San Francisco, CA, USA, 1966. 